\title{
Analysis of Network Management Technology and Development Trend In The Future
}

\author{
Lishen Yang \\ Department of Computer Science and Technology. \\ Henan Polytechnical University \\ Jiaozuo, China \\ yangls@hpu.edu.cn
}

\author{
Qiang Wang \\ Department of Computer Science and Technology. \\ Henan Polytechnical University \\ Jiaozuo, China \\ cuky17potato@yahoo.com.cn
}

\begin{abstract}
This paper introduces the typical network management technology and some hot technology. The trend of network management development in the future is prospected based on the analysis of the current network management technology.
\end{abstract}

Keywords-network management technology; data acquisition; distributed network management; SNMP

\section{INTRODUCTION}

Since the computer network appears, the scale and complexity of the earlier computer network is not so large, but along with the development of computer technology, the computer network becomes more and more popular, and the role it plays is more and more important. With the rapid development of network technology, people are increasingly dependent on the network, and simple network connectivity is not able to meet the requirements of users already. They are requiring the special network that has faster speed, better quality and safety. However, the continually increasing number of the network users will raise more and more difficult problems to daily management and maintenance of the network. In order to keep the increasingly larger-scale network systems contributing normally, ensure that all network source in good running condition, corresponding network management system is needed to provide the control function. Therefore, how to operate the management of the network effectively has become a growing problem[1].

On the other hand, along with the increasing complexity of the computer network, the demand towards the network performance is much higher while the unprecedented development opportunity to the network management will be brought. In order to meet the demand of network application, the hierarchical, integrated and intelligent will be the development trend of modern network management technology. Network management protocols are enriched constantly, and the application of CMIP (Common Management Information Protocol) is also expanding gradually, starting to take on the more complex tasks of network management. Therefore, technical innovation in network management system appears rather important particularly, further, only the technology continues to be improved can make the network management system in rapid development.

\section{Hot Technology In Network Management}

Many issues that cared about by the network management technician, such as installation and configurations, resource sharing, backup and restore, system security and performance optimization, are all important aspects of modern network management. In this situation, the traditional, highly centralized network management model cannot meet the need of complex network management. In terms of the management function requirements of the network management software, the hot technology has the following several aspects:

\section{A. Technology of data acquistion}

In the aspect of data acquisition and processing, the network management function will be achieved basically using polling and trap technology. However, with the increasing number of network users and the unceasingly strengthening network complexity, the total amount of data that needs to be collected is getting huger and the demand on the performance of the network management is increasingly higher as well as the network transmission quality. Currently, the traditional data acquisition technology cannot deal with this situation well. In order to break through this bottleneck, the mobile agent acquisition technology is brought up. Not only the mobile agent technology has the characteristics of independent, initiative, interaction and cooperation that ordinary agent owns, but also it can be achieved that the migration will be accomplished among the different hosts without ending programs. It is because of the high intelligent and portability that can reduce the amount of data transferred in the network and remove the intermediate step of data processing, so that the load of management station can be reduced. For the management platform, the mobile agent with transparency, good portability, flexible programming ability and rich extension function can be used in the management of large scale distributed heterogeneous network[2].

\section{B. Technology of data management and display}

The remote network equipment can be managed only from the administration console with the traditional network management technology, as well as the collected data information can be operated. In order to implement a more convenient and intuitive way to do the network management that the administrator with the different permission level can 
view and manage the data in any place of the world, that the data information collected is more simple and intuitively presented, the concepts including WBM technology, Portal technology and Xml technology are introduced[3].

\section{1) Network management based on Web}

WBM (Web-Based Management) technology assembles the advantages of the two technologies, Web function and network management technology, so that the stronger operation ability than what the traditional tools can supply for the network administrator could be provided. WBM technology allows administrator configure, visit, control network and other parts on any network node rapidly with any web browser. This technology is a revolution towards the network management, which will improve a further step to the way of network management.

The network management model based on Web can be achieved in two ways which are non-interference between them. The first way is the agent model. This combines a Web server with an internal workstation which takes turns to communicate with terminal equipment. The browser users set up a communication channel with agent via HTTP protocol while agent set up a communication channel with terminal equipment via SNMP protocol (Fig. 1). The other way to realize the WBM technology is the embedded system model. In this model, the web property finally can be embedded into network devices and each device has its own IP address. The administrator can access and manage the devices through the browser easily (Fig. 2).

These two methods have their respective advantages and both of them will be continually used to operate the network management together, other than replacing each other[1]. The agent model not only retains the advantages of the network management system based on the workstation, but also adds the advantages of access flexibility and mobility enhancement. The embedded system model could bring in the graphical management technology which ensures the interface can be easily used and it is better than the command line currently as well as the remote login interface based on menu.

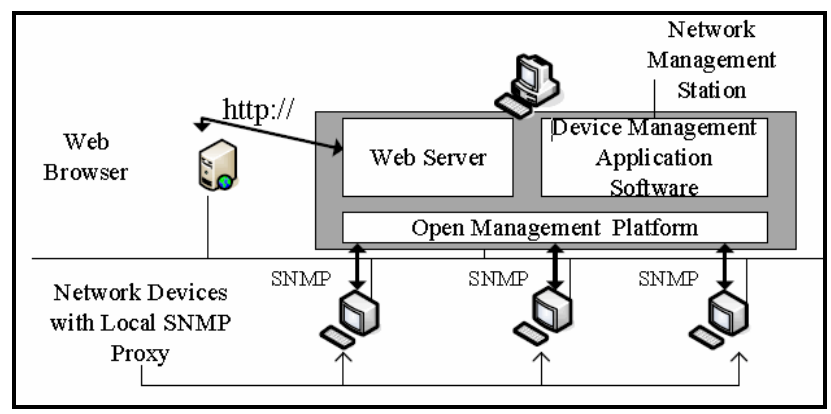

Figure 1. Proxy model

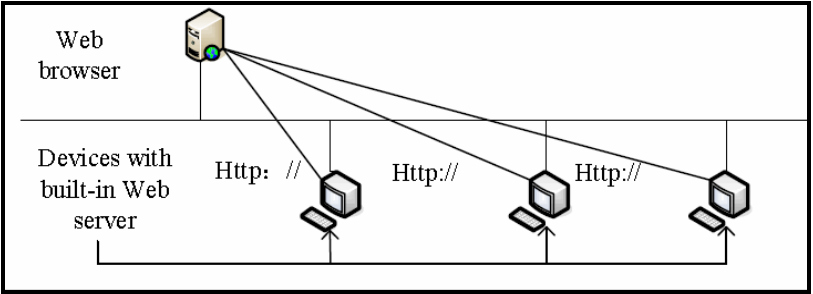

Figure 2. Embedded system model

\section{2) Portal technology}

Portal technology is an important trend as the development of the Web application. It will be a unified platform of a variety of application system resource, data resource, information resource under the network and roles, the personalized application interface will be established, and this model will link different users through the processing, transmission to the event and message. This way is full of strong expansibility, compatibility and comprehensive. Therefore, as the Web technology has been applied to the network management today, it will not be far away that application of Portal technology in network management level.

\section{3) XML technology}

XML (Extensible Markup Language) technology is an international standard, which can effectively unify the existing multiple management interface in network system. The system provides a standard source of information, which will be described with a way that easy to read and understand among the different users and systems[5]. Therefore, the network application has better interoperability, universality and expansibility. And this technology has great flexibility, by which the embedded management agent in heterogeneous network equipment can be controlled. It also ensures the reliability of interactive communication and operation between the management system and network devices as well as among the different management system. Due to a simple markup language, which is easy to be realized in management system development and integration process, has been applied to XML technology itself, this new management interface can be implemented to lower the cost of the management system development.

\section{THE DEVELOPMENT TREND OF NETWORK MANAGEMENT}

The traditional network management system always focuses on the model that the various heterogeneous network equipments in the network layer are managed and controlled through simple network management protocol. However, along with the network user continually increasing, the topological structure complexity of the network continually increasing, as well as the user applications that will require the increasing network bandwidth are brought in, nowadays, the network management technology begins to permeate to application layer, facing the probable issues in the future. Certain amount of these applications dependences on realtime, such as real-time voice and video data transmission 
while other ones need the normal data transmission rate. Therefore, in order to achieve the higher network bandwidth utilization rate, the fast and reliable service (QOS) could be provided respectively to the various applications according to the differences among the application service. Currently, the focus of the network management technology begins to be transferred to application layer from network layer, which is a breakthrough in network management system[6].

Note that, the network management presents its diversity gradually, but associated with kinds of standardized protocol emergence and interconnection of heterogeneous network, the network management development are still has the trends below:

\section{A. Implementation of Distributed Network Management}

Distributed network management model could implement network management function through the interaction between the network management client distributed physically and the network management server. This model could distribute the function of information management and intelligent judgment throughout the whole network, which means that the basic fault treatment decisions could be made at the problem source or closer to the fault source at least, making the management become more automatic. Distributed network management will set up multiple domain management workstations that are used to be responsible for the managed devices that in their respective domains, meanwhile, coordination and interaction are also run among the domain workstations at the same time, to complete the whole network management. It not only reduces load of the core network management server, but also as far as to avoid the network resource occupancy that the network management information result in, improving the efficiency. Distributed network management model is shown in Fig. 3.

The present distributed technology is mainly discussed from two aspects: one is the technology named CORBA (Common Object Request Broker Architecture) which is still in the research stage and another is the mobile agent technology. The mobile agent technology is still discussed in individual fields and the period when this technology could be brought into the network management field is still unknown. The future network management model will be the combination of the centralized and distributed management model. The distributed management model could meet the requirement of the task that consuming

memory and occupying bandwidth, such as data collection. Therefore, this model could reduce the load of the management workstation and weaken the network congestion[7].

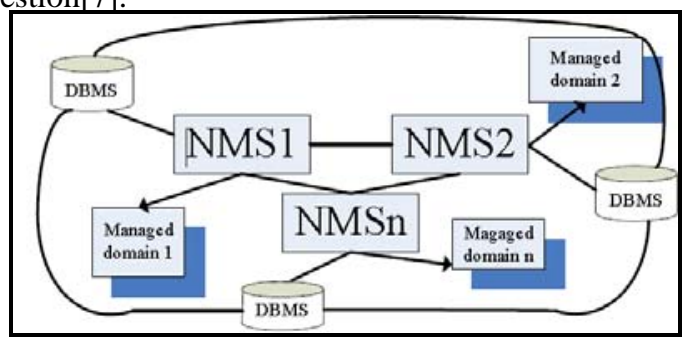

Figure 3. Distributed network management model

\section{B. Implementation of Intergrated Network Management}

The integrated network management requires the management support to the multiple models. The perspective to each sub network, the knowledge to the managed business as well as the fault location and removal can be implemented by the operation to a console center, which means realizing the management to the multiple network that are interactions. With the rapid development of the network appear the different types of network, such as SDH (Synchronous Digital Hierarchy) network, IP network, and among these ones exists different degrees of connectivity[1]. And there are also a variety of network management systems that come from different manufactures, comply with different standards and respectively manage their own devices, increasing the difficulty of the network management. Therefore, currently, the integrated network management system could be realized in two ways: one is that according to the situation of each sub management system, the integrated network management can be established to implement the management to each sub network management system; the other is to set up directly the integrated network management system using the existing technology.

\section{Implementation of Intelligent Network Management}

Along with the rapid development of modern computer network management, the maintenance of the network management is becoming more and more complex and the higher requirement has been raised to the network administrator. At the same time, artificial management to the network is often time-consuming and laborious, but also the intermittent cannot be timely eliminated. So intelligent is the development trend of network management software in the future, and improve work efficiency really embodies the function of network management software. Intelligent network management software should be able to obtain a variety of network equipment technical parameters automatically, and then continue to implement intelligent analysis, diagnosis, and early warning. Fault diagnosis and automatic network maintenance, which can be used to explain the error information of network operation, fault diagnosis and provide treatment recommendations, is the earliest network management field that artificial intelligence is applied in. Performance expert system will be able to analyze the operating parameters and data, and the network failure can be predicted and troubleshooting before found by user. In addition, intelligent network management system has the strong pre-fault processing function that is able to run fault recovery automatically, making every effort to guarantee the reliability of the network.

\section{Implementation of Security Network Management}

Network information service embodies the value of the network, and many security problems will involved in the usage and management of network information process. Such as: computer virus attacks, vulnerability of internet/Intranet internal security, man-made malicious 
attacks and security vulnerability of network operating system. Therefore, how to guarantee the security of network information service and management is very important. Safety is the support of the network, so the security of network management software is one of hot technologies. In addition to the security mechanism of software itself, currently, SNMP protocol has been applied in much network management software, commonly SNMPvl, SNMPv2, but at this stage, the SNMPvl, SNMPv2 protocol for safety control is relatively weak, raising a challenge to the development of follow-up SNMP protocol [9]

\section{CONCLUSION}

With the continuous development of network technology, it will ceaselessly make forward progresses with the application of various new technologies, so as to provide convenient, fast and effective management to numerous networks. However, the present computer network management function is only the realization of the part function of the network management system development and application. There is still a certain gap from the whole realization of the modern network management system and we can do the further research and development in this area, in order to make this management perfect.

\section{ACKNOWLEDGMENT}

I must thank Professor Yang. He has afforded such better support to my paper, from when the topic was determined till it was finished. And then I need to give my many thanks to my classmates for their care and support all the time. Without their kindly help, I can never finish my paper in time.

\section{REFERENCES}

[1] Zhuang Wu, "The Principle and Realization of Computer Network Management,” Electronic Industry Press, pp. 15-20, 2009.

[2] Xudong $\mathrm{Hu}$, Hong $\mathrm{Yu}$ and Ying Chen, "Web-based Data Acquisition,” Journal of Zhejiang University Science, Vol. 3, 2002.

[3] Jiahai Yang, Xiankun Ren and Peiyu Wang, "Technology of Network Management and Realization,” Tsinghua University Press, pp. 83-89, 2000.

[4] Haitao Xia, Zhiqiang Zhan, "A New Generation of Network Management Technology,” Beijing University of Posts and Telecommunications Press, pp. 45-47, 2003.

[5] Gongxuan Zhang, Yongfeng Ling and Jianfang Ge, "The data Manipulation Technology on XML," Journal of Manjing University of Science and Technolgy, Vol. 27, 2003

[6] Xiaoyu Tong, Lu Liu and Luying Li, “A New Generation Theory and Technology of Mobile Fusion Network," Posts and Telecommunications Press, pp. 101-103. 2012.

[7] Mingyu Shen, Yousheng Zhang, "Distributed Intelligent Management Model Based on Active Network,” Pattern Recognition and Artificial intelligence, Vol. 21, 2008

[8] Jun Guo, Feng Liu and Xudong Chen, "Design and Implement of SDH Intergrated Network Management System,” Microcomputer \& Its Application, Vol.23, 2004

[9] Xingwei Wang, “A New Generation of Internet Technology and Application”, Higher Education Press, pp. 56-61. 2011. 